\title{
Exactly solvable model of a slightly fluctuating ratchet
}

\author{
V. M. Rozenbaum, ${ }^{1, *}$ T. Ye. Korochkova, ${ }^{1}$ I. V. Shapochkina, ${ }^{2}$ and L. I. Trakhtenberg ${ }^{3}$
}

${ }^{1}$ Chuiko Institute of Surface Chemistry, National Academy of Sciences of Ukraine, Generala Naumova str. 17, Kiev 03164, Ukraine

${ }_{2}$ Department of Physics, Belarusian State University, Prospekt Nezavisimosti 4, Minsk 220050, Belarus

3 Semenov Federal Research Center of Chemical Physics, Russian Academy of Sciences, Kosygin Street 4, Moscow 119991, Russia; Moscow Institute of Physics and Technology, Institutsky Lane 9, Dolgoprudny 141700, Moscow Region, Russia; and Lomonosov Moscow State University, 1-3 Leninskie gory, Moscow, 119991, Russia

(Received 23 March 2021; revised 1 June 2021; accepted 24 June 2021; published 26 July 2021)

\begin{abstract}
We consider the motion of a Brownian particle in a sawtooth potential dichotomously modulated by a spatially harmonic perturbation. An explicit expression for the Laplace transform of the Green function of an extremely asymmetric sawtooth potential is obtained. With this result, within the approximation of small potential-energy fluctuations, the integration of the relations for the average particle velocity is performed in elementary terms. The obtained analytical result, its high-temperature, low-frequency, and high-frequency asymptotics, as well as numerical calculations performed for a sawtooth potential of an arbitrary symmetry, indicate that in such a system, the frequency-temperature controlling the magnitude and direction of the ratchet velocity becomes possible. We clarify the mechanism of the appearance of additional regions of nonmonotonicity in the frequency dependence of the average velocity, which leads to the appearance of additional ratchet stopping points. This mechanism is a consequence of the competition between the sliding time along the steep slope of the highly asymmetric sawtooth potential and the correlation time of the dichotomous noise.
\end{abstract}

DOI: 10.1103/PhysRevE.104.014133 